\title{
Results from the MSGC tracker at SMC
}

M.K. Ballintijn a , F.D. van den Berga, R. van Dantziga, G. Gracia ${ }^{b}$, N. de Groot ${ }^{a}$, F.G. Hartjes $^{a}$, R. Horisberger ${ }^{c}$, D. Kaandorp ${ }^{a}$, T. J. Ketel ${ }^{a}$, M.F. Litmaath ${ }^{a}$, J.J. Niessink ${ }^{a}$, A. Ogawa $^{d}$, E.P. Sichtermann ${ }^{a}$, F. Udo ${ }^{a}$ and A.R. de Winter ${ }^{a}$

${ }^{a}$ NIKHEF, PB 41882, NL1009DB Amsterdam, The Netherlands

${ }^{b}$ GAES, University of Santiago de Compostella, Spain

cPSI, Villigen, Switzerland

dNagoya University, Japan

January 1995
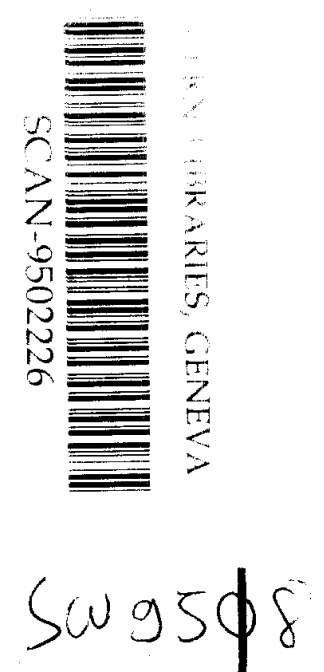

Talk given by F.G. Hartjes at the 4th International Conference on Advanced Technology and Particle Physics, Como (Italy), 3 - 7 October 1994 


\section{Results from the MSGC tracker at SMC}

M.K. Ballintijn ${ }^{a}$, F.D. van den Berga, R. van Dantziga , G. Gracia ${ }^{b}$, N. de Groot ${ }^{a}$, F.G. Harties $^{a}$, R. Horisberger ${ }^{c}$, D. Kaandorp ${ }^{a}$, T. J. Ketela, M.F. Litmaath ${ }^{a}$, J.J. Niessink ${ }^{a}$, A. Ogawa $^{\mathrm{d}}$, E.P. Sichtermann ${ }^{\mathrm{a}}$, F. Udo ${ }^{\mathrm{a}}$ and A.R. de Winter ${ }^{\mathrm{a}}$

aNIKHEF, PB 41882, NL1009DB Amsterdam, The Netherlands

bGAES, University of Santiago de Compostella, Spain

cPSI, Villigen, Switzerland

dNagoya University, Japan

A tracker consisting of 16 MSGCs has been installed in the high intensity muon beam of the SMC experiment[1] at CERN. Each MSGC has an active surface of $10 \times 10 \mathrm{~cm}^{2}$, covered by 496 anode strips. As a front-end amplifier the APC 64 is used.

Results are presented about the efficiency, both at a high rate and at a low rate, and the position resolution. Using the data of the MSGC tracker the definition of the beam tracks in SMC significantly improved.

\section{Introduction}

The SMC experiment at CERN (NA47) measures the spin structure of the nucleon. A $190 \mathrm{GeV}$ muon beam is scattered on a twin target of polarised frozen butanol. The two target halves are polarised in opposite directions. It is important to have a good determination of

\begin{abstract}
the event vertex down to very small scattering angles. To achieve this, two systems of MicroStrip Gas Counters (MSGCs) have been installed. They improve the measurement of the position and direction of both the incoming and the scattered muon. In this paper we describe the set-up of the tracker and present first results of its functioning.
\end{abstract}

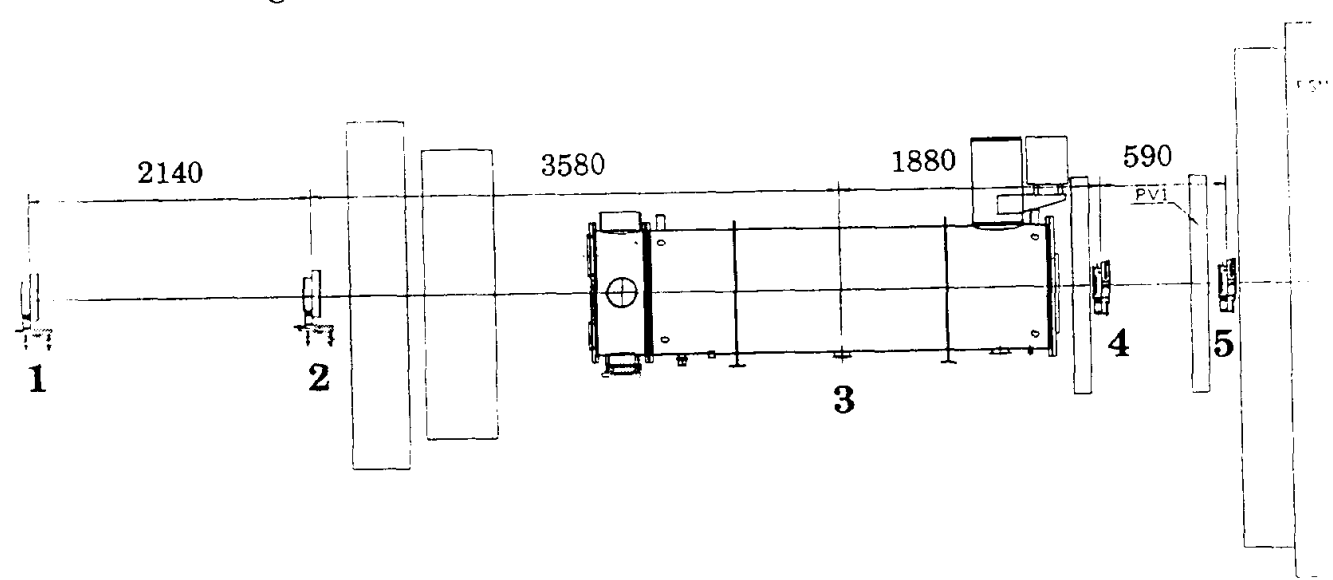

Figure 1. Part of the SMC set-up where 1 and 2 are the stations of the upstream telescope, 3 is the polarised target and 4 and 5 are the stations of the downstream telescope. The distances are in $\mathrm{mm}$. 


\section{Set-up of the MSGC tracker}

The tracker is composed of 16 MSGCs in 8 gastight boxes. In each box two MSGC planes are placed under an angle of $90^{\circ}$ with respect to each other. Two boxes are mounted back to back under an angle of $45^{\circ}$ to form a station. The upstream telescope (fig. 1) consists of two stations in front of the SMC target to measure the incident particle. The downstream telescope is formed by a second set of two stations at the other side of the target to measure the particles exiting at small angles. To minimise multiple scattering of the tracks at bigger angles, the wall thickness of the aluminium boxes of the downstream telescope is only $2 \mathrm{~mm}$.

The parameters of the MSGC substrate are given in table 1 . It is glued onto a printed circuit board together with the front-end electronics. The delicate strip pattern and the input of the front-end electronics are protected against sparks in two ways: the cathode strips are connected, in groups of 16 , by an SMD resistor in the $M \Omega$ range to the $H V$ supply thus reducing the electrical energy that is released in case of a spark to $20 \mu \mathrm{J}$. In addition the peak current in the preamp is limited to less than $1 \mathrm{~A}$ by a resistor between the anode strip and the preamp. The resistors are made from a NiCr layer deposited on a $2 \mathrm{~mm}$ wide glass strip.

Table 1

MSGC substrate

material

size D263 glass

metalisation width anode strips width cath.strips $300 \mu \mathrm{m} \times 4 " \mathrm{x} 4 "$ $1 \mu \mathrm{m}$ aluminium $7 \mu \mathrm{m}$

$90 \mu \mathrm{m}$ anode pitch
Table 2

Spark protection

\begin{tabular}{ll}
\hline & \\
\hline anode resistor & \\
\hline value & $740 \Omega$ \\
metalisation & $5 \mathrm{~nm} \mathrm{NiCr}$ \\
surface & $8 \times 10^{4} \mu \mathrm{m}^{2}$ \\
& \\
cath. resistor & $4.7 \mathrm{M} \Omega$ \\
\hline value & 16 \\
\hline \# of grouped cath. & \\
\hline
\end{tabular}

The sensitive area of each MSGC plane is sufficiently large to cover the full beam profile having a width of $\sigma=12 \mathrm{~mm}$. The charge collection time amounts to 50 ns.

Table 3

MSGC parameters

\begin{tabular}{ll} 
sensitive area & $93 \times 99 \mathrm{~mm}^{2}$ \\
gas gap & $2.7 \mathrm{~mm}$ \\
\# of APCs & 8 \\
\# of anode ch. & 496 \\
\hline
\end{tabular}

\subsection{Front-end electronics}

As a front-end preamplifier the APC $64[2]$ is used, a device consisting of 64 integrators, each followed by a 32 element switched capacitor pipeline. Using double correlated sampling, only the difference between the signal level at a sample point before and after the event is sequentially read out. The uncorrelated electronic noise amounts to $1200 \mathrm{NEC}$ at $\mathrm{C}_{\mathrm{in}}=0 \mathrm{pF}$. Since this number is dominated by the internal switching noise of the APC, it could be reduced by almost a factor of two by reading out two neighbouring capacitors, instead of one, for each sample point.

The eight APCs of an MSGC all showed an equal common mode noise about as big as the uncorrelated noise, which was not easy to suppress. To compensate for this, the average of the first and the last eight channels was taken as a baseline reference. Note that the first and last eight electronic channels of each MSGC were not connected to an 
anode strip. The noise distribution is not purely Gaussian; the tails are suppressed. This means that a lower threshold can be applied. We applied a threshold of 6000 electrons, which is about four times the RMS of the uncorrelated noise.

The time between the two sample points defines the integration time. However, due to the long rise time of the preamplifier the smallest time window is still $300 \mathrm{~ns}$ as is shown in fig. 2 .

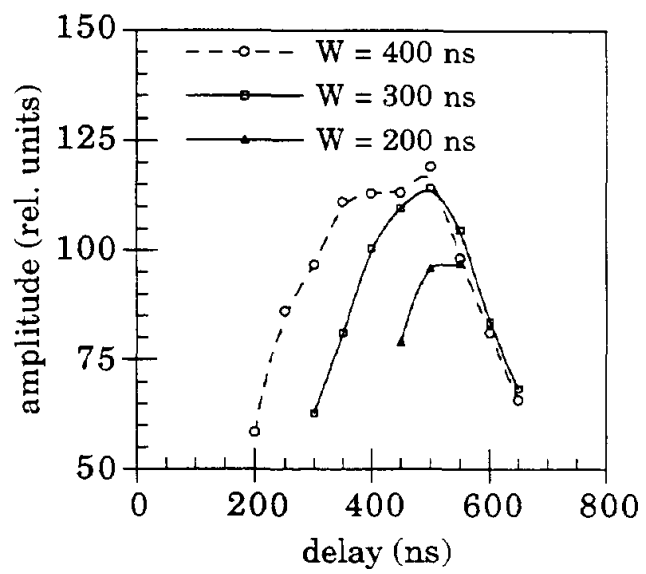

Figure 2. APC amplitude as a function of the trigger delay for three different time intervals (W) between the sample points.

The 16 APCs of two MSGC planes are read-out sequentially by a Fastbus Sirocco and added to the data stream of the experiment.

Table 4

Front-end electronics

type

sample frequency

APC64, SAC2

read out frequency

$12 \mathrm{MHz}$

$1.5 \mathrm{MH}$

power consumption $\quad<300 \mu \mathrm{W} / \mathrm{ch}$

\subsection{Experimental conditions}

The applied counter gas matches a good HV stability with a low diffusion, a high primary ionisation and a low $y$ sensitivity [3].

Table 5

Operating conditions

counter gas $\quad \mathrm{DME} / \mathrm{CO}_{2} 60 / 40$

drift field $\quad 7 \mathrm{kV} / \mathrm{cm}$

cath. strip voltage $\quad-600$ to $-630 \mathrm{~V}$

gas gain 640

prim. ionisation $\quad 50$ electrons

thresh. upstream 12 prim.electrons

thresh. downstr. 22 prim.electrons

beam rate $\quad 20 \mathrm{MHz}$

signal current $\quad 70 \mathrm{nA}$

Due to the high beam rate and the broad time window of the APC, several tracks pass through the detector for each trigger. They often have a lower pulse height, but still high enough to pass the threshold. To prevent overflow of the buffers in the online system and to assure reliable off-line track reconstruction, it was decided to raise threshold, thus sacrificing on efficiency.

\section{Results}

\subsection{Position resolution}

From fig. 3 we derived a position resolution for a single layer of $54 \mu \mathrm{m}$. However, this value is deteriorated by $2 \%$ broken anodes. Measurements have shown that most of the ionisation arriving near a not connected anode will not get lost, but will be captured instead by the nearest properly functioning anode strip. Therefore, a broken anode will not lead to loss of efficiency, but instead to an increase of the position error. The measured value of $54 \mu \mathrm{m}$ is thus expected to go down if the effect from the known interrupted anodes is taken into account. 


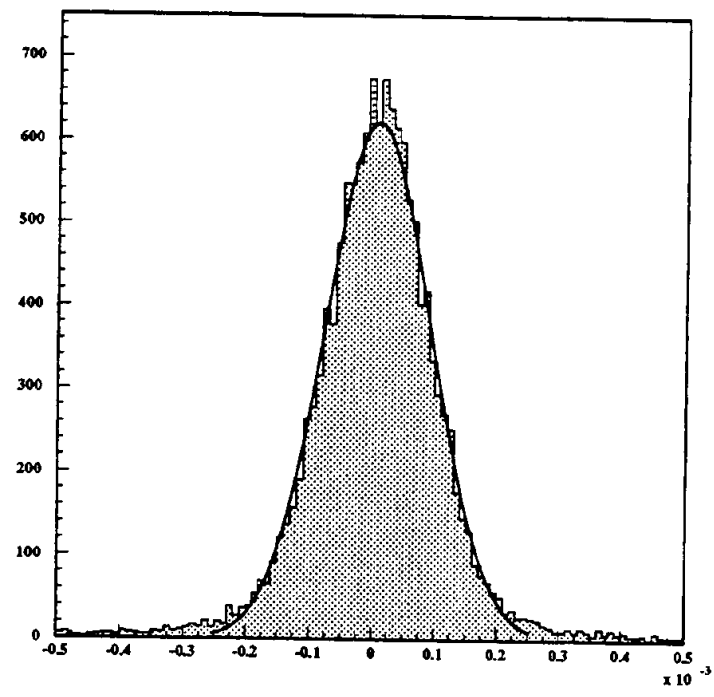

Figure 3. Residuals of tracks fitted through all four MSGC planes of a station.

\subsection{Efficiency}

The efficiency in the downstream telescope for an individual MSGC is plotted for various distances to the centre of the beam in fig. 4 .

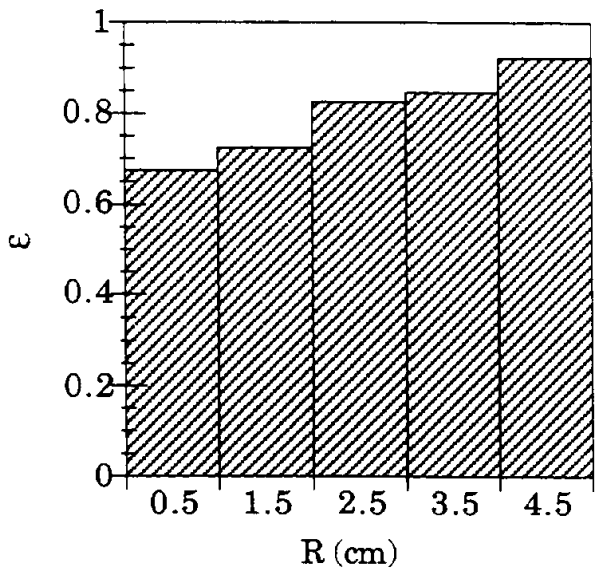

Figure 4. Efficiency $(\varepsilon)$ for various distances (R) from the beam centre
The figure shows that while at the edge the efficiency exceeds $90 \%$, at the centre it has already dropped down to less than $70 \%$. Two effects play a role in this phenomenon: 1 . the regular rate dependence of an MSGC using D263 glass and 2 . the increased rate dependence by ageing. The first effect is caused by ions from the avalanche that stick to the glass surface and thus modify the electrical field in the vicinity of the anode. From experiments at NIKHEF (fig. 5) we found a decrease of $20 \%$ in the amplitude by this effect. The second effect is caused by the MSGC ageing.

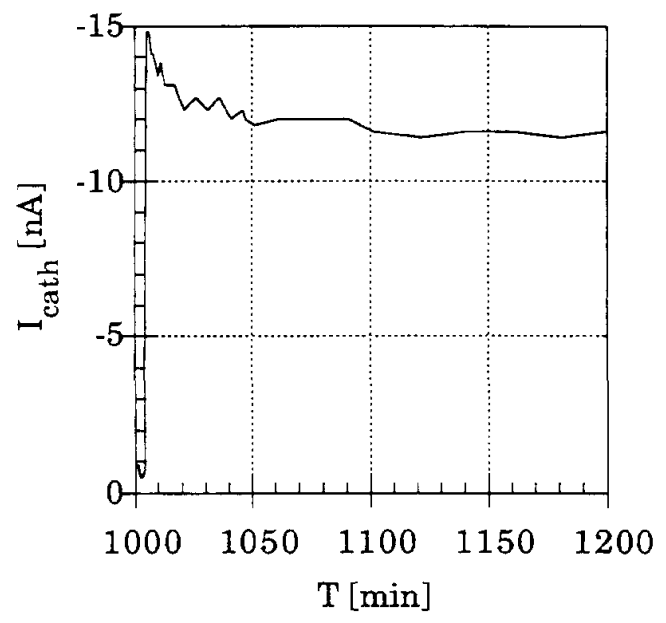

Figure 5. Result of a laboratory test showing the decrease of the gas gain (here indicated by $I_{\text {cath }}$ ) after the start of the irradiation.

\subsection{Ageing}

From the number of data-taking runs elapsed since the installation of the MSGCs, the chamber current during the burst and the measured beam profile, the integrated charge collected at the centre of the beam was calculated as $500 \mu \mathrm{C} / \mathrm{cm}$. Measurements done earlier at NIKHEF where an MSGC on D263 glass was 
irradiated by a ${ }^{90} \mathrm{Sr}$ source showed a significant drop of the gas gain (fig. 6).

Examination of the substrate learned that the effect is probably caused by the voltage drop across a thin transparent layer of a polymer formed on top of the anode. Using this information, we would expect for the $500 \mu \mathrm{C} / \mathrm{cm}$ of collected anode charge in the case of SMC an estimated thickness of the polymer layer of $500 \mathrm{~nm}$, affecting the gain seriously. However, since the polymer also has a certain conductivity, the voltage drop will depend upon the electrical current through the layer and thus upon the particle rate. Therefore, in practice the observed ageing will show up as a pronounced rate sensitivity.

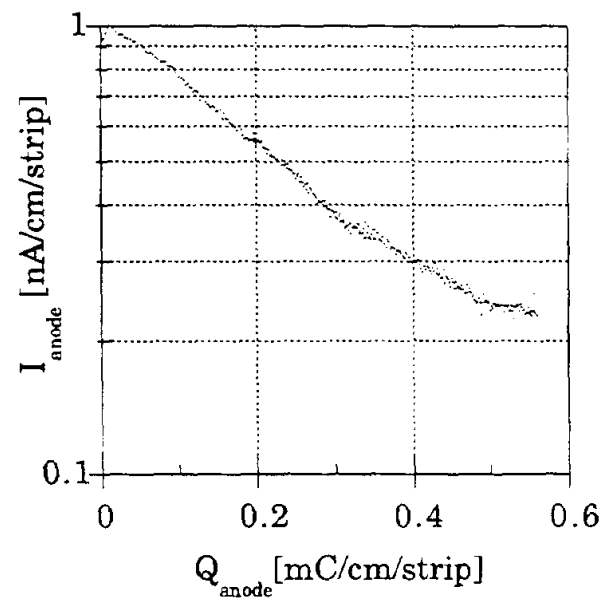

Figure 6. Laboratory measurement of the gain (here indicated by $\mathrm{I}_{\text {cath }}$ ) as a function of the integrated charge on the anode strip of an MSGC by ageing.

A more direct indication of the ageing is obtained from the curves of the cluster amplitude that are deduced from the SMC data. A cluster is defined as a group of adjacent strips whose signal exceeds the threshold. The cluster amplitude is the sum of the pulse heights of the cluster strips.

Fig. 7 shows the different cluster amplitudes for tracks in the centre of the beam and those outside. It clearly can be seen that the threshold cuts away tracks with a low amplitude and therefore reduces the efficiency. A Landau fit of both curves shows that the amplitude in the centre is $64 \%$ of the one in the outside regions. The laboratory measurements (fig. 5 and 6) indicate a drop to $25 \%$ at the centre of the beam for the combined effect of the regular rate dependence and the ageing. This is not in contradiction with the amplitude measured by SMC, which includes the average over an area with a particle rate between the extreme values.

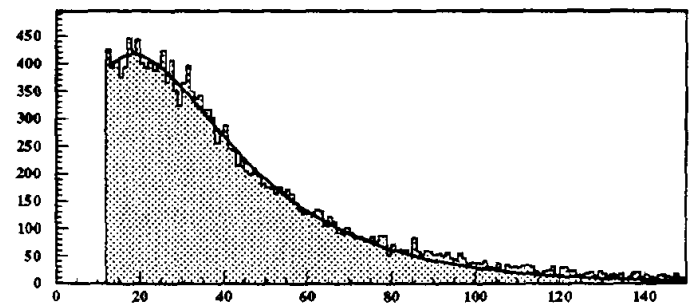

Figure 7a. Cluster amplitude in the centre of the beam $(R \leq 2 \mathrm{~cm})$

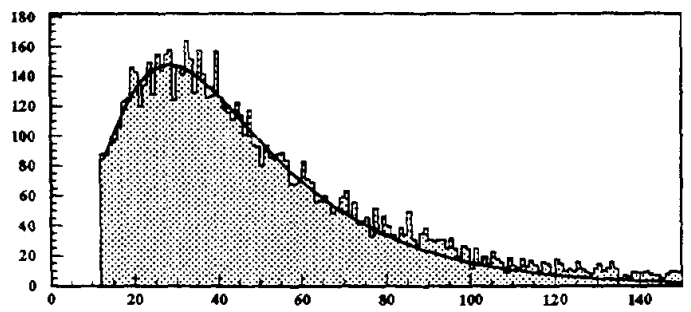

Figure $7 \mathrm{~b}$. Cluster amplitude outside the centre $(2<R \leq 4 \mathrm{~cm})$.

\subsection{Performance of the MSGC tracker}

The MSGCs are used to improve the track resolution in the off-line reconstruction. A minimum of 4 out of the 
8 planes of a telescope is required to be included in the track fit. The upstream telescope could be operated very efficiently and was included in more than $95 \%$ of the beam tracks. The downstream telescope had to be operated at a higher threshold, and was less efficient. About $60 \%$ of the scattered tracks were improved by the MSGC measurements.

Both the position resolution and the angular accuracy of the tracks measured by the MSGCs improved by a factor of two. This resulted in a gain of about $5 \%$ of correctly reconstructed events at small angles (< $10 \mathrm{mrad}$ ). This number is expected to go up slightly when the fine tuning of the alignment and the calibration is done. Further, for all angles the error on the vertex position is reduced, for the highest angles up to a factor of 2 . The error on the vertex position is now dominated by multiple scattering in the target.

Another positive effect of the MSGCs was that it made it possible to do a very precise alignment of the other chambers and to measure temperature effects.

\section{Conclusions}

The MSGCs have been successfully integrated in the SMC experiment. A good result has been obtained for the position measurement. This can be improved further by correcting for broken anode strips. The error on the track position and direction has been decreased by a factor of two.

The ageing of the MSGCs has been measured and found compatible with laboratory results. Due to this ageing and the high threshold, the efficiency of the MSGCs in the centre of the beam is relatively low.

Most problems in the data are due to the long integration time that had to be used because of the long rise time of the preamplifier. This problem will hopefully be solved by using an updated version of the APC64 in 1995

\section{References}

[1] Spin Muon Collaboration, B. Adeva et al., Phys. Lett. B 329 (1994) 399.

[2] R. Horisberger and D. Pitzl, Nucl. Instr. and Meth. A 326 (1993) 92.

[3] M.H.J. Geijsberts et al., Nucl. Instr. and Meth. A 315 (1992) 529. 